PROCEEDINGS OF THE

AMERICAN MATHEMATICAL SOCIETY

Volume 33, Number 2, June 1972

\title{
THE CONVERGENCE DETERMINING CLASS OF CONNECTED OPEN SETS IN PRODUCT SPACES
}

\author{
DIETER LANDERS
}

\begin{abstract}
It is proved in this paper that each sequence of measures with values in a topological group-defined on the Borel field of a finite or countable product of connected, locally connected, separable merric spaces-which is Cauchy convergent for all connected open sets is Cauchy convergent for all Borel sets, too.
\end{abstract}

1. Preliminaries. In this paper a topological group $G$ is always assumed to be abelian. The system of neighborhoods of the zero element of $G$ is denoted by $\mathscr{U}(0)$. A sequence $a_{n} \in G, n \in N$, is Cauchy convergent iff it is Cauchy convergent with respect to the uniformity

$$
\{\{(a, b) \in G \times G: a-b \in U\}: U \in \mathscr{U}(0)\} .
$$

Let $\mathscr{B}$ be a $\sigma$-field on $X$; a function $\mu: \mathscr{B} \rightarrow G$ is a measure iff for all sequences of disjoint sets $A_{i} \in \mathscr{B}, i \in N$, the sequence $\left(\sum_{i=1}^{n} \mu\left(A_{i}\right)\right)_{n \in N}$ converges to $\mu\left(\sum_{i \in N} A_{i}\right)$.

Let $\mathscr{B}_{0}$ be a subsystem of $\mathscr{B}, A \in \mathscr{B}$, and $\mu: \mathscr{B} \rightarrow G$ be a measure. We write $\mathscr{B}_{0} \cap A=\left\{B \cap A: B \in \mathscr{B}_{n}\right\}$ and $\mu\left(\mathscr{B}_{0} \cap A\right)=\left\{\mu(B): B \in \mathscr{B}_{0} \cap A\right\} \subset G$. The set $N:=\bigcap\{U: U \in \mathscr{U}(0)\}$ is a closed subgroup of $G$. The quotient group $G / N$ is a Hausdorff group and the natural homomorphism $\pi: G \rightarrow$ $G / N$ is continuous, open and $\pi^{-1} \pi G_{0}=G_{0}+N$ for each $G_{0} \subset G$. Replacing $G$ by $G / N$ we may assume for our purpose without loss of generality that $G$ is a Hausdorff group.

If $X$ is a topological space we denote by $\mathscr{T}_{X}$ the system of open sets in $X$ and by $\mathscr{B}_{X}$ the Borel field of $X$, i.e. the $\sigma$-field generated by $\mathscr{T}_{X}$. If $Z=X \times Y$ and $A \subset Z, \pi_{X} A:=\{x \in X:(x, y) \in A$ for some $y \in Y\}$ denotes the projection of $A$ to $X . \bar{A}$ denotes the complement of a set $A$.

2. The main results. It is well known that convergence of (probability) measures for all connected open sets of the space $\boldsymbol{R}$ of real numbers does not imply convergence for all Borel sets (let e.g. $\left.P_{n}(\{n\})=1, n \in N\right)$. The following result shows, however, that this becomes true for all product

Received by the editors September 30, 1971.

AMS 1970 subject classifications. Primary 60B10; Secondary 28A35.

Key words and phrases. Convergence of measures, connected open sets, product space, topological group.

c American Mathematical Society 1972 
spaces $\boldsymbol{R}^{n}$ with $n \geqq 2$. It turns out that the proof heavily relies on the fact that these spaces are product spaces. More specifically the proof works for every finite or countable product of connected, locally connected, separable metric spaces.

THEOREM 1. Let $Z$ be the product space of two connected, locally connected, separable metric spaces $X, Y$ containing at least two elements. If a sequence of measures defined on the Borel field of $Z$ with values in $a$ topological group is Cauchy convergent for all connected open sets of $Z$, then it is Cauchy convergent for all Borel sets of $Z$.

Proof. Let $G$ be a topological group and assume that $\mu_{n}, n \in N$, is a sequence of measure defined on the Borel field $\mathscr{B}_{Z}$ with values in $G$ which is Cauchy convergent for all connected open sets.

(i) At first we shall show: For each closed $U \in \mathscr{U}(0)$ and each nonvoid $B \in \mathscr{T}_{\boldsymbol{F}}$ there exists a nonvoid open, connected set $B_{0} \subset B$ such that $\mu_{n}\left(\mathscr{B}_{Z} \cap\left(X \times B_{0}\right)\right) \subset U$ for all $n \in N$ :

Since $Y$ is a connected, locally connected metric space containing at least two elements, there exist nonvoid disjoint open and connected sets $B_{i} \subset B, i \in N$. Then the sets $X \times B_{i}, i \in N$, are disjoint, open and connected. We shall show that for some $i \in N, \mu_{n}\left(\mathscr{B}_{Z} \cap\left(X \times B_{i}\right)\right) \subset U$ for all $n \in N$. Assume conversely that for each $i \in N$ there exists $n(i) \in N$ with $\mu_{n(i)}\left(\mathscr{B}_{Z} \cap\left(X \times B_{i}\right)\right) \notin U$. Then $n(i) \rightarrow \infty$ because otherwise there exists $n_{0} \in N$ such that $\mu_{n_{0}}\left(\mathscr{B}_{Z} \cap\left(X \times B_{i}\right)\right) \notin U$ for infinitely many $i \in N$; Lemma 5, however, implies

$$
\mu_{n_{0}}\left(\mathscr{B}_{Z} \cap\left(X \times B_{i}\right)\right) \subset \mu_{n_{0}}\left(\mathscr{B}_{Z} \cap \sum_{n \geqq i}\left(X \times B_{n}\right)\right) \subset U
$$

for all sufficiently large $i \in N$. Let $v_{i}:=\mu_{n(i)}, i \in N$. Let $U_{1} \in \mathscr{U}(0)$ be symmetric such that $U_{1}+U_{1} \subset U$. Then by Lemma 4 for each $i \in N$ there exists an open and connected set $C_{i} \subset X \times B_{i}$ with $\pi_{X}\left(C_{i}\right)=X$ and $\nu_{i}\left(C_{i}\right) \notin U_{1}$. We remark that $C_{i}, i \in N$, are disjoint sets. Now we shall show that

(1) $\left(v_{n}\left(\sum_{i \in M} C_{i}\right)\right)_{n \in N}$ is Cauchy convergent for each $M \subset N$.

Let $A_{1}, A_{2} \subset X$ be nonvoid disjoint open and connected sets. Let $M \subset N$ be given and define $D_{i}:=\left(A_{i} \times Y\right) \cup \sum_{j \in M} C_{j}, D_{12}:=\left(A_{1} \times Y\right) \cup$ $\left(A_{2} \times Y\right) \cup \sum_{j \in M} C_{j}, E_{i}:=D_{i}-\left(A_{i} \times Y\right)$ and

$$
E_{12}:=D_{12}-\left(\left(A_{1} \times Y\right) \cup\left(A_{2} \times Y\right)\right) \quad(i=1,2) .
$$

As $A_{1} \times Y, A_{2} \times Y, D_{1}, D_{2}$ and $D_{12}$ are open and connected and $\left(v_{n}\right)_{n \in N}$ is Cauchy convergent for all connected open sets, $\left(v_{n}\right)_{n \in N}$ is Cauchy convergent for $E_{1}, E_{2}$ and $E_{12}$ and hence for $\sum_{j \in M} C_{j}=E_{1}+\left(E_{2}-E_{12}\right)$.

Now (1) implies, according to Lemma $6, \lim _{i \in N} v_{i}\left(C_{i}\right)=0$, which yields a contradiction. 
(ii) We shall show that $\left(\mu_{n}\right)_{n \in N}$ is Cauchy convergent for all open sets of $Z$.

Let $V$ be an open set in $X \times Y$. Since $X \times Y$ is locally connected and has a countable base, there exists at most a countable number of components of $V$. say $V_{n} \in \mathscr{T}_{Z}, n \in M \subset N$. Let a closed $U_{0} \in \mathscr{U}(0)$ be given and $U_{n} \in \mathscr{U}(0)$, $n \in N$, be closed and symmetric such that $U_{n}+U_{n} \subset U_{n-1}, n \in N$. According to (i), applied for each $i \in M$ to $B:=\pi_{Y}\left(V_{i}\right)$, there exist nonvoid open and connected sets $B_{i} \subset \pi_{Y}\left(V_{i}\right)$ such that $\mu_{n}\left(\mathscr{B}_{Z} \cap\left(X \times B_{i}\right)\right) \subset U_{i+3}$ for all $n \in N$. Furthermore we obtain by (i) a nonvoid open and connected set $A \subset X$ such that $\mu_{n}\left(\mathscr{B}_{Z} \cap(A \times Y)\right) \subset U_{3}$ for all $n \in N$.

Let $C:=V \cup(A \times Y) \cup \bigcup_{i \in M}\left(X \times B_{i}\right)$. Then $C$ is an open and connected set in $X \times Y$ and

$$
\mu_{n}(C)=\mu_{n}(V)+\mu_{n}\left(\bar{R} \cap\left((A \times Y) \cup \bigcup_{i \in M}\left(X \times B_{i}\right)\right)\right) \in \mu_{n}(V)+U_{2},
$$

whence $\mu_{n}(V) \in \mu_{n}(C)+U_{2}$ for all $n \in N$. As $\left(\mu_{n}(C)\right)_{n \in N}$ is Cauchy convergent, $\mu_{n}(C)-\mu_{m}(C) \in U_{1}$ for all sufficiently large $n, m \in N$. Hence

$$
\mu_{n}(V)-\mu_{m}(V) \in \mu_{n}(C)-\mu_{m}(C)+U_{2}+U_{2} \subset U_{1}+U_{2}+U_{2} \subset U_{0}
$$

for all sufficiently large $n, m \in N$. This implies (ii).

(iii) As each sequence of measures defined on the Borel field of a metric space with values in a topological group is Cauchy convergent for all Borel sets if it is Cauchy convergent for all open sets [2, Corollary 5], (ii) implies the assertion.

Corollary 2. Let I be an at most countable index set with $|I| \geqq 2$. Let $X_{i}, i \in I$, be connected, locally connected and separabie metric spaces such that $\left|X_{i}\right| \geqq 2$ for at least two $i \in l$. If a sequence of measures defined on the Borel field of $\mathrm{X}_{i \in I} X_{i}$ with values in a topological group is Cauchy convergent for all connected open sets, then it is Cauchy convergent for all Borel sets, too.

Proof. Let $i_{0} \in I$ be such that $\left|X_{i_{0}}\right| \geqq 2$. Then $Y:=\mathrm{X}_{i \in I-i_{0}} X_{i}$ is connected, locally connected, separable metric and contains at least two elements. Hence the assertion follows from Theorem 1, applied to $X:=X_{i_{0}}$ and $Y$.

COROLLARY 3. Let $X$ be a connected, locally connected and separable metric space. If a sequence of measures defined on the Borel field of $X^{\mathbf{N}}$ $\left[X^{n}\right.$ for $\left.n \geqq 2\right]$ with values in a topological group is Cauchy convergent for all connected open sets, it is Cauchy convergent for all Borel sets, too.

ProOF. Follows immediately from Corollary 2.

We remark that Corollary 3 is applicable to separable normed spaces and hence especially to the space of real numbers. 


\section{Auxiliary lemmas.}

Lemma 4. Let $Z$ be a product space of two connected, locally connected separable metric spaces $X, Y$ containing at least two elements. Let $\mu$ be a measure defined on the Borel field $\mathscr{B}_{Z}$ of $Z$ with values in a topological group $G$. Let furthermore $U, U_{1} \in \mathbb{l}(0)$ with $U_{1}+U_{1} \subset U, U$ closed, $U_{1}$ symmetric, and let $B \subset Y$ be an open and connected set such that $\mu\left(\mathscr{B}_{Z} \cap(X \times B)\right) \notin U$. Then there exists an open and connected set $C \subset$ $X \times B$ with $\pi_{X} C=X$ and $\mu(C) \notin U_{1}$.

Proof. (i) There exists an open set $T \subset X \times B$ such that $\mu(T) \notin U$.

Since $\mu\left(\mathscr{B}_{Z} \cap(X \times B)\right) \notin U$, there exists $D \in \mathscr{B}_{Z}, D \subset X \times B$ with $\mu(D) \notin U$. As $\bar{U}$ is open and $\mu(D) \in \bar{U}$ there exists $V \in \mathscr{U}(0)$ with $\mu(D)+V \subset \bar{U}$. Since each measure on a metric space with values in $G$ is closed approximable (see [2, Proposition 15]) there exists a closed set $F \subset \bar{D}$ with

$$
\mu\left(\mathscr{B}_{Z} \cap(\bar{D}-F)\right)=\mu\left(\mathscr{B}_{Z} \cap(F-D)\right) \subset V .
$$

Let $T:=F \cap(X \times B)$. Then $T \subset X \times B$ is open and $\mu(T)=\mu(D)+$ $\mu(T-D) \in \mu(D)+V \subset \bar{U}$, i.e. $\mu(T) \notin U$.

(ii) If $\varnothing \neq A \subset X$ is open, then for each $V \in \mathscr{U}(0)$ there exists a nonvoid open and connected set $A_{0} \subset A$ with $\mu\left(\mathscr{B}_{Z} \cap\left(A_{0} \times Y\right)\right) \subset V$.

Since $X$ is a connected, locally connected metric space containing at least two elements, there exist nonvoid disjoint open and connected sets $A_{i} \subset A, i \in N$. Then $A_{i} \times Y, i \in N$, are disjoint sets, whence (ii) follows from Lemma 5.

(iii) Let $U_{n+1} \in \mathscr{U}(0), n \in N$, be symmetric such that $U_{n+1}+U_{n+1} \subset U_{n}$ for all $n \in N$. As the assumptions on $X$ and $Y$ are symmetric, by (ii) there exists a nonvoid open and connected $B_{0} \subset B$ such that $\mu\left(\mathscr{B}_{Z} \cap\left(X \times B_{0}\right)\right) \subset$ $U_{2}$. Since $X \times Y$ is locally connected and has a countable base, the open set $T \subset X \times Y$ of (i) contains at most a countable number of components, say $T_{n} \in \mathscr{T}_{Z}, n \in M \subset N$. As $\pi_{X}\left(T_{n}\right)$ is open, for each $n \in M$ there exists by (ii) a nonvoid open and connected set $A_{n} \subset \pi_{X}\left(T_{n}\right)$ such that

$$
\mu\left(\mathscr{B}_{Z} \cap\left(A_{n} \times Y\right)\right) \subset U_{n+2} \text {. }
$$

Let $C:=T \cup\left(X \times B_{0}\right) \cup \cup_{n \in .1 I}\left(A_{n} \times B\right)$. Then $C \subset X \times B$ is open and connected. As $\mu(T) \notin U$ and $\mu\left(\mathscr{B}_{Z} \cap\left(\left(X \times B_{0}\right) \cup \bigcup_{n \in M}\left(A_{n} \times B\right)\right)\right) \subset U_{2}+U_{2} \subset U_{1}$, we obtain $\mu(C) \notin U_{1}$.

For the sake of completeness we cite the following two lemmas, which are proved in [1] (respectively [2]).

Lemma 5. Let $\mu$ be a measure defined on a $\sigma$-field $\mathscr{B}$ with values in a topological group $G$. If $A_{n} \in \mathscr{B}, n \in N$, decreases to $\varnothing$ then for every $U \in \mathscr{U}(0), \mu\left(\mathscr{B} \cap A_{n}\right) \subset U$ for all sufficiently large $n \in N$.

Proof. See Lemma 12 of [2]. 
LEMMA 6. Let $\mu_{n}, n \in N$, be a sequence of measures, defined on a $\sigma$ field $\mathscr{B}$ with values in a topological group. If $A_{k} \in \mathscr{B}, k \in N$, are disjoint sets such that $\left(\sum_{k \in M} \mu_{n}\left(A_{k}\right)\right)_{n \in N}$ is Cauchy convergent for each $M \subset N$, then $\lim _{n \in N} \mu_{n}\left(A_{n}\right)=0$.

Proof. Apply Lemma 10(2) of [1] to $b_{i, k}:=\mu_{i}\left(A_{k}\right), 1, k \in N$.

ACKNOWLEDGEMENT. The author wishes to thank Mr. L. Rogge for valuable suggestions.

\section{REFERENCES}

1. D. Landers and L. Rogge, The Hahn-Vitali-Saks and the uniform-boundedness theorem in topological groups, Manuscripta Math. 4 (1971), 351-359.

2. - Cauchy convergent sequences of regular measure with values in a topological group, Z. Wahrscheinlichkeitstheorie und Verw. Gebiete 21 (1972), 188-196.

Mathematisches Institut, University of Cologne, Cologne, Federal Republic of Germany 